\section{Comparison of dynamic contour tonometry and noncontact tonometry in ocular hypertension and glaucoma}

M Erdurmus, Y Totan, IF Hepsen and R Yagci

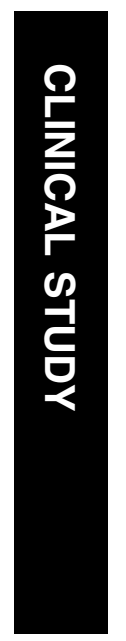

measurements, did not depend on corneal thickness.

Eye (2009) 23, 663-668; doi:10.1038/eye.2008.3; published online 8 February 2008

Keywords: tonometry; intraocular pressure; corneal thickness; primary open-angle glaucoma; ocular hypertension

\section{Introduction}

Glaucoma is the leading cause of irreversible vision loss worldwide and is of major public health concern, leading to loss of mobility and independence. $^{1-3}$ Intraocular pressure (IOP) has a critical role in case detection and management of primary open-angle glaucoma (POAG). ${ }^{4}$ IOP reduction is currently the only treatment available for decreasing the risk of glaucoma progression. Ocular hypertension (OHT) is associated with an increased risk of developing glaucoma, ${ }^{5}$ and reducing IOP has been shown to lessen progressive loss of the visual field. ${ }^{6}$ Therefore, determination of real IOP is critical for the management of glaucoma.

Applanation tonometry is the method of measuring IOP with instruments that flatten the corneal apex. Goldmann applanation tonometry (GAT) is a widely established and the most common indirect method for identifying IOP for the last four decades and is the gold standard for measuring the IOP. ${ }^{7-11}$ GAT, while being minimally invasive, still requires the instillation of fluorescein, ocular topical anaesthesia, and corneal contact. In addition to this, there are some advantages of the other objective instruments, such as noncontact tonometers
Department of

Ophthalmology, Fatih University Medical School, Ankara, Turkey

Correspondence:

M Erdurmus,

Department of

Ophthalmology,

Fatih University Medical

School,

Alparslan Turkes Cad.

No. 57,

Emek,

Ankara 6510,

Turkey

Tel: + 90312 2126262;

Fax: +903122213276

E-mail: merdurmus@

yahoo.com

Received: 25 April 2007 Accepted in revised form: 19 December 2007 Published online: 8 February 2008

Financial interest: None 
(NCTs), over the GAT. The NCT has the potential advantage that it uses an air puff to applanate the cornea, reducing the possible risk of cross-infection with agents such as adenovirus and variant Creutzfeldt-Jakob disease. ${ }^{12,13}$ Corneal anaesthesia and direct corneal contact are not required to obtain an IOP reading compared with GAT. Thus, NCTs are comfortable for the patient with a minimal risk of infection, in addition to being an easy to use and rapid method of IOP measurement. Recently, usage of NCTs in diagnosis and management of glaucoma has been gradually increasing in many countries. ${ }^{4}$

A new digital, slit-lamp-mounted tonometer has been recently introduced as an alternative to applanation tonometry. The dynamic contour tonometer (DCT) (Pascal tonometer, Swiss Microtechnology AG, Port, Switzerland) is a nonapplanation contact tonometer designed to be largely independent of the corneal biomechanical properties. The medical-grade silicon tip of DCT creates a tight-fitting shell with the cornea when in contact with it and compensates for all forces exerted on it. A pressure sensor that is centrally and concavely embedded into the tonometer tip measures the IOP transcorneally. DCT has been reported to be unaffected by central corneal thickness (CCT) in healthy and glaucoma subjects ${ }^{14-16}$ and in refractive surgery patients before and after laser in situ keratomileusis as well. ${ }^{17,18}$ Therefore, it is important to ascertain the agreement of NCT with DCT along with reproducibility of DCT for the assessment of IOP in POAG and OHT subjects.

The aim of this study was to assess the agreement in the measurement of IOP obtained by DCT and NCT in patients with POAG and OHT and to investigate the relationship between CCT and IOP measurements using both instruments. The second goal of this study was to assess interobserver and intraobserver reproducibility of IOP measurement by means of DCT.

\section{Materials and methods}

\section{Study population}

Patients with POAG and OHT were included in the study. The local university ethics committee approved the study and the tenets of the Declaration of Helsinki were observed. Written informed consent was obtained from all the participants.

\section{Agreement of DCT and NCT IOP readings}

One hundred and four patients with POAG $(n=75)$ or OHT $(n=29)$ were included in this prospective study. POAG was defined as glaucomatous optic nerve damage, glaucomatous visual field defect, IOP of $22 \mathrm{~mm} \mathrm{Hg}$ or beyond, and an open anterior chamber angle in gonioscopy. OHT was defined as IOP of $22 \mathrm{~mm} \mathrm{Hg}$ or more in the presence of a normal optic nerve head, normal visual field, and normal gonioscopy. Exclusion criteria were as follows: history of intraocular inflammation or trauma; previous or current corneal disease; contact lens wear; subjects with astigmatism greater than 3.0 dioptres (D); and any ocular surgery, including anterior segment laser applications. To minimize systematic bias, only the left eye of each patient was included in the study. Prior to IOP measurements, the cornea was anaesthetized with eye drops (proparacain). During the measurement, subjects were asked to keep both eyes open, breathe quietly, and fixate into the distance behind the examiner. After patients had given informed consent, IOP was measured in random order (to allow for changes in IOP produced by applanation of the cornea) by the NCT and DCT with 5 min intervals.

The mean of three consecutive measurements of NCT readings was recorded. The same experienced examiner took DCT readings. Quality score $(\mathrm{Q})$ of DCT readings is classified from Q1 (optimum) to Q5 (unacceptable) by the manufacturer. Q4 and Q5 measurements, which indicate poor data quality, were excluded from the study. Although Q3 is classified as 'acceptable' by the manufacturer, intraobserver variability is higher than for $\mathrm{Q} 1$ and Q2. Thus, the mean of three consecutive Q3 measurements was calculated or in cases of high-quality measurements (Q1 and Q2), one reading was considered sufficient. ${ }^{19}$

Subsequently, CCT was determined by an ultrasonic pachymeter (Echo Scan US-80, Nidek, Tokyo, Japan). The pachymeter probe was placed on the centre of the cornea over an undilated pupil and the mean of three readings within an SD of $\pm 5 \mu \mathrm{m}$ was calculated for each eye.

\section{Reproducibility of DCT and NCT}

The left eyes of another 41 patients with POAG $(n=30)$ or OHT $(n=11)$ with age ranging from 41 to 82 years were chosen for the reproducibility study. Patients with a history of intraocular inflammation or trauma, previous or current corneal disease, contact lens wear, with astigmatism greater than $3.0 \mathrm{D}$, and any ocular surgery including anterior segment laser applications were excluded from the study. All IOP measurements were performed with an undilated pupil. In this study, estimation of IOP was not commenced until each of the examiners had completed a learning period (at least 10-15 patients) with the DCT. Two operators (IFH and $\mathrm{ME}$ ) independently measured the IOP by means of DCT 
and NCT as described previously in a random sequence with 5 min intervals, with three IOP measurements each. All IOP measurements were obtained at $30 \mathrm{~s}$ intervals within the devices. An assistant recorded each measurement. The observers were masked to all IOP measurements.

\section{Statistical analysis}

Variables showed almost perfect normal distribution in both Kolmogorov-Smirnov test and Q-Q plots. SPSS (statistical software) 11.5 for Windows was used for the calculation of means, SDs, paired sample $t$-test, linear regression analysis, analysis of variance (ANOVA), coefficient of repeatability, and variance components estimation. $P$-value smaller than 0.05 was considered significant. We used the Bland-Altman graph for analysing the amount of agreement between NCT and DCT IOP readings. MedCalc Version 7.4.2.0 (MedCalc Software, Mariakerke, Belgium) program was used for the Bland-Altman analysis.

Intraobserver reproducibility was based on the analysis of two independent series of measurements made by the two examiners (six examinations).

Reproducibility was evaluated by means of the (two-way random, consistency) coefficient of repeatability. The systematic difference between methods was termed the 'bias' and random differences were quantified by the 'limits of agreement'. Where there was no relation between interinstrument or interobserver differences and IOP magnitude, bias was calculated as the mean difference and $95 \%$ limits of agreement were computed (provided that the differences followed a normal distribution).

\section{Results}

The age, sex, IOP readings with NCT and DCT, CCT, and the mean difference of IOP measurements between tonometers are shown in Table 1.

Table 1 Age and sex, IOP readings with NCT and DCT, CCT, and the mean difference of IOP measurements between tonometers

\begin{tabular}{lcc}
\hline Parameters & Mean $\pm S D$ (range) & P-value $^{\mathrm{a}}$ \\
\hline Age (years) & $61.4 \pm 12.8(25-92)$ & \\
Sex $($ female $/$ male $)$ & $50 / 54$ & \\
NCT $(\mathrm{mm} \mathrm{Hg})$ & $19.2 \pm 4.4(9-30)$ & \\
DCT $(\mathrm{mm} \mathrm{Hg})$ & $20.0 \pm 3.6(12.9-30.7)$ & \\
NCT-DCT $(\mathrm{mm} \mathrm{Hg})$ & $-0.8 \pm 3.0$ & 0.009 \\
CCT $(\mu \mathrm{m})$ & $563 \pm 45(475-663)$ & \\
\hline
\end{tabular}

$\mathrm{CCT}$, central corneal thickness; DCT, dynamic contour tonometer; IOP, intraocular pressure; $\mathrm{NCT}$, noncontact tonometer; $\mathrm{SD}$, standard deviation. aPaired sample $t$-test.

\section{Agreement of DCT and NCT IOP readings}

Figure 1 shows a Bland-Altman plot of NCT and DCT differences against the average of NCT and DCT values. The mean difference (95\% limits of agreement) between NCT and DCT was $-0.80 \pm 2.98$ ( -6.6 to 5.1$) \mathrm{mm} \mathrm{Hg}$, and no relation between NCT/DCT differences and average was found. The $95 \%$ confidence interval (CI) for bias of mean NCT/DCT difference was -1.57 to $-0.03 \mathrm{~mm} \mathrm{Hg}$. The $95 \%$ CI for the bias of lower and upper agreement limits were -7.70 to -5.50 and $4.40-6.20$, respectively.

\section{Effect of CCT and age on NCT and DCT IOP measurements}

The IOP readings obtained by NCT depended on CCT $\left(P=0.000\right.$; adjusted $r^{2}=0.301 ; 95 \%$ CI 0.038-0.070; a slope of $0.054 \mathrm{~mm} \mathrm{Hg}$ per $1 \mu \mathrm{m} \mathrm{CCT)} \mathrm{by} \mathrm{linear} \mathrm{regression}$ analysis. In contrast to NCT, DCT readings showed no effect of CCT $\left(P=0.388\right.$; adjusted $r^{2}=-0.002 ; 95 \% \mathrm{CI}$ -0.009 to 0.023$)$. The difference between NCT and DCT readings was also affected by CCT $(P<0.001$; adjusted $r^{2}=0.518$; 95\% CI 0.039-0.056; a slope of $0.047 \mathrm{~mm} \mathrm{Hg}$ per $1 \mu \mathrm{m} \mathrm{CCT).} \mathrm{Scatter} \mathrm{plot} \mathrm{of} \mathrm{CCT} \mathrm{against} \mathrm{IOP} \mathrm{difference}$ between NCT and DCT is shown in Figure 2.

Linear regression analysis on DCT and NCT readings showed no effect of age $(P=0.985,95 \% \mathrm{CI}-0.056$ to 0.057 , adjusted $r^{2}=-0.01 ; P=0.121,95 \% \mathrm{CI}-0.121$ to 0.014 , adjusted $r^{2}=0.014$, respectively).

All patients with POAG $(n=75)$ were treated with monotherapy or with combined topical therapy. In the OHT group, 13 of 29 patients were treated with

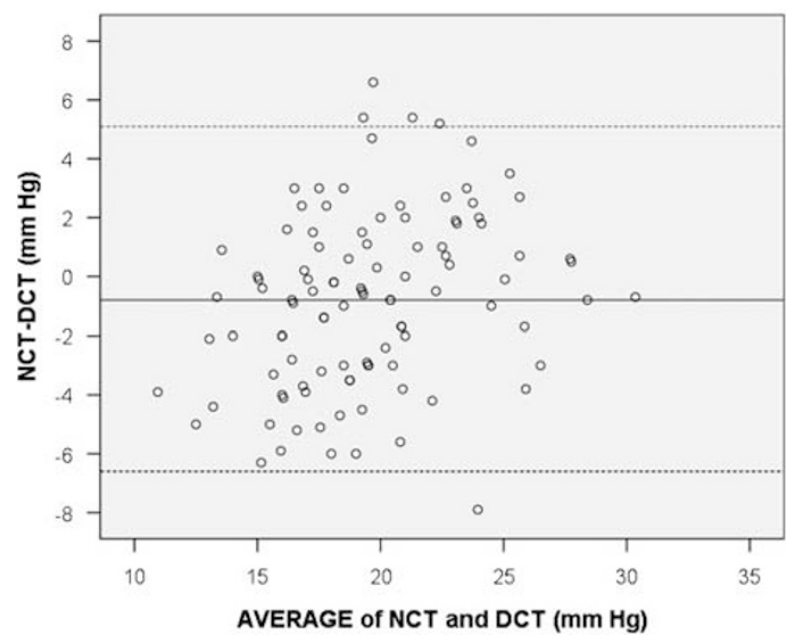

Figure 1 Bland-Altman plots of noncontact tonometer (NCT)/dynamic contour tonometer (DCT) intraocular pressure (IOP) differences against NCT and DCT mean. There was no relation between NCT and DCT readings (Outer lines indicate $\pm 1.96 \mathrm{SD}$ ). 


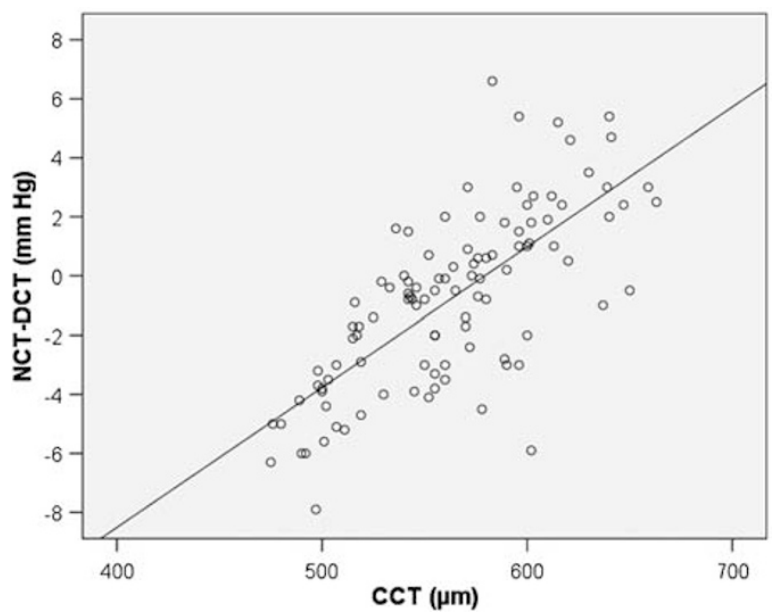

Figure 2 Scatter plots of central corneal thickness (CCT) against intraocular pressure (IOP) differences between noncontact tonometer (NCT) and dynamic contour tonometer (DCT).

monotherapy. In the treated group $(n=88)$, mean $\mathrm{NCT} / \mathrm{DCT}$ difference was $-1.2 \pm 2.9 \mathrm{~mm} \mathrm{Hg}$. In the untreated group $(n=16)$, mean NCT/DCT difference was $1.4 \pm 2.3 \mathrm{~mm} \mathrm{Hg}$. Analysis of variance (ANOVA) performed to test whether treatment status had an effect on NCT/DCT differences showed statistically significant effect $(P=0.001)$.

\section{Intraobserver and interobserver reproducibility}

The descriptive statistics for each observer are summarized in Table 2. No difference was found between the IOP readings taken in the three sessions for both examiners by means of DCT and NCT $(P>0.05$ for both). For this reason, the average of the three readings was used for the interobserver variability study. The coefficient of repeatability was 0.92 (95\% CI $0.85-0.96$, $P<0.001)$ for DCT and 0.95 (95\% CI 0.91-0.97, $P<0.001$ ) for NCT.

There were no statistically significant interobserver differences between mean NCT and DCT measurements ( $P>0.05$ for both). The mean interinstrument difference between NCT and DCT was $0.02 \pm 1.04(-0.31$ to 0.34$)$ and it was not statistically significant $(P=0.9)$.

\section{Discussion}

In this study, we compared DCT with NCT, a widely used tonometer in ophthalmology clinics, and evaluated the effect of CCT on IOP measurements with both tonometers. We found no agreement between the IOP readings obtained by both tonometers and no effect of corneal thickness on DCT readings $(P=0.388)$ over a wide range of CCT. In contrast to DCT, IOP readings
Table 2 Descriptive statistics of the intraocular pressure measurements $(\mathrm{mm} \mathrm{Hg})$ by means of dynamic contour tonometer and noncontact tonometer performed by the two examiners

\begin{tabular}{lccccc}
\hline Examiner & \multicolumn{2}{c}{ DCT } & & \multicolumn{2}{c}{ NCT } \\
\cline { 2 - 3 } & Mean & SD & & Mean & $S D$ \\
\hline IFH1 & 19.3 & 4.4 & & 19.2 & 4.5 \\
IFH2 & 18.8 & 4.7 & & 18.9 & 4.6 \\
IFH3 & 18.8 & 4.5 & & 19.0 & 4.4 \\
IFH mean & 19.0 & 4.5 & & 19.0 & 4.5 \\
ME1 & 19.9 & 4.4 & & 19.2 & 4.4 \\
ME2 & 19.0 & 4.1 & & 19.5 & 4.6 \\
ME3 & 18.8 & 4.0 & & 18.9 & 4.2 \\
ME mean & 19.1 & 4.1 & 19.2 & 4.4 \\
IFH mean -ME mean & -0.2 & 1.7 & -0.2 & 1.4 \\
\hline
\end{tabular}

$\mathrm{SD}$, standard deviation.

obtained by NCT were dependent on CCT $(P<0.001)$, in that NCT measurements showed a linear correlation with an increase in IOP of $0.054 \mathrm{~mm} \mathrm{Hg}$ per $1 \mu \mathrm{m} \mathrm{CCT}$. We also investigated the intraobserver and interobserver reproducibility of IOP measurements by DCT. The analyses of intraobserver and interobserver variability by means of DCT seem to be highly reproducible.

Ogbuehi ${ }^{20}$ reported no significant difference in intersession repeatability indices between GAT and Topcon CT-80 NCT and the average IOP measured by both tonometers. He found that the mean difference in IOP measurements between the two techniques was $0.2 \pm 1.5 \mathrm{~mm} \mathrm{Hg}$ and the $95 \%$ limits of agreement were -3.14 and $+2.74 \mathrm{~mm} \mathrm{Hg}$. These limits of agreement show good relation between GAT and CT-80 NCT IOP readings. Therefore, he suggested that NCT is an accurate and reliable method for assessing IOP and that its IOP readings are interchangeable with those measured with the GAT. Nevertheless, this study underwent IOP range of $9-21 \mathrm{~mm} \mathrm{Hg}$ in healthy individuals. Therefore, there is no clear evidence that the agreement can be generalized to IOP values that are not in this range.

Goldmann ${ }^{21}$ speculated that the tonometers accuracy might be questionable in corneas with a CCT outside a normal range. Now the effect of CCT on the accuracy of IOP measurements with different tonometers is well known. ${ }^{14,22-24}$ The CCT has become an important biometric factor and is an essential part of the evaluation of glaucoma. Siganos et $a l^{17}$ reported that GAT and NCT readings significantly correlated with CCT, and the mean change in IOP readings was approximately $3 \mathrm{~mm} \mathrm{Hg}$ for every $100 \mu \mathrm{m}$ change in CCT. They also found that DCT was not influenced by CCT. A manometry experiment showed that measurement with DCT provides IOP values significantly closer to true manometric levels than either GAT or pneumotonometer. ${ }^{25}$ Francis et $a l^{26}$ 
reported that DCT was less affected than GAT by variations in CCT in 2157 participants of primarily Mexican ancestry. We found that CCT showed no significant effect on DCT readings in the present study, which corresponds well with previous studies. ${ }^{14,17,26-28}$ IOP measured by NCT, on the other hand, was dependent on CCT. We found that in eyes with POAG and OHT, the NCT readings correlated significantly with CCT, and our findings indicate a slope of $0.054 \mathrm{~mm} \mathrm{Hg}$ per $1 \mu \mathrm{m}$ increase in CCT. Possible explanation for these differences in IOP change per $100 \mu \mathrm{m}$ change in CCT in different studies may be related to patient factors (eg race and diagnosis of POAG and OHT) and unknown factors influencing IOP, such as corneal hydration state and corneal rigidity.

The 95\% agreement limits of the NCT and DCT are wider than reported in a previous study, which compared GAT and DCT, where limits from -1.6 to $2.4 \mathrm{~mm} \mathrm{Hg}$ were found. ${ }^{25}$ Some other studies showed wider 95\% agreement limits between DCT and GAT. ${ }^{15,29}$ Although we did not find any relation between IOP measurements by DCT and those by NCT, a wide range of $95 \%$ agreement limits may partly be related with the wide range of CCT in the subjects studied.

This study demonstrated that in common with other tonometers such as GAT and NCT, measurement of IOP using the DCT seems to indicate that both the intraobserver and interobserver reproducibilities of IOP measurements are extremely high. The coefficient of repeatability was 0.92 , which compared favourably with that obtained by previous studies. ${ }^{14,15}$ The results of our study suggest that the relatively simple, highly reproducible, and objective nature of DCT should allow any well-trained operator to make highly reliable IOP measurements.

In conclusion, DCT presents a new technology of noninvasive IOP measurement. The DCT showed no agreement when compared with NCT in patients with POAG and OHT. In the subjects studied, IOP measurements with DCT did not depend on CCT. DCT may offer some clinically relevant advantages over conventional NCTs for screening and management of POAG or OHT and a potential clinical role for subjects with CCT outside the normal range.

\section{References}

1 Quigley HA. Number of people with glaucoma worldwide. Br J Ophthalmol 1996; 80: 389-393.

2 Evans JR, Fletcher AE, Wormald RP. MRC Trial of Assessment and Management of Older People in the Community. Causes of visual impairment in people aged 75 years and older in Britain: an add-on study to the MRC Trial of Assessment and Management of Older People in the Community. Br J Ophthalmol 2004; 88: 365-370.

3 Ramrattan RS, Wolfs RC, Panda-Jonas S, Jonas JB, Bakker D, Pols HA et al. Prevalence and causes of visual field loss in the elderly and associations with impairment in daily functioning: the Rotterdam Study. Arch Ophthalmol 2001; 119: 1788-1794.

4 Tonnu PA, Ho T, Sharma K, White E, Bunce C, GarwayHeath D. A comparison of four methods of tonometry: method agreement and interobserver variability. $\mathrm{Br} J$ Ophthalmol 2005; 89: 847-850.

5 Gordon MO, Beiser JA, Brandt JD, Heuer DK, Higginbotham EJ, Johnson CA et al. The ocular hypertension treatment study: baseline factors that predict the onset of primary open-angle glaucoma. Arch Ophthalmol 2002; 120: 714-720.

6 Heijl A, Leske MC, Bengtsson B, Hyman L, Bengtsson B, Hussein M, Early Manifest Glaucoma Trial Group.

Reduction of intraocular pressure and glaucoma progression: results from the Early Manifest Glaucoma Trial. Arch Ophthalmol 2002; 120: 1268-1279.

7 Brandt JD. Corneal thickness in glaucoma screening, diagnosis, and management. Curr Opin Ophthalmol 2004; 15: 85-89.

8 Dielemans I, Vingerling JR, Hofman A, Grobbee DE, de Jong PT. Reliability of intraocular pressure measurement with the Goldmann applanation tonometer in epidemiological studies. Graefes Arch Clin Exp Ophthalmol 1994; 232: 141-144.

9 Goldmann H, Schmidt T. [Applanation tonometry]. Ophthalmologica 1957; 134: 221-242.

10 Wessels IF, Oh Y. Tonometer utilization, accuracy, and calibration under field conditions. Arch Ophthalmol 1990; 108: 1709-1712.

11 Whitacre MM, Stein R. Sources of error with use of Goldmann-type tonometers. Surv Ophthalmol 1993; 38: 1-30.

12 Walia JS, Chronister CL. Possible iatrogenic transmission of Creutzfeldt-Jakob disease via tonometer tips: a review of the literature. Optometry 2001; 72: 649-652.

13 Desai SP, Sivakumar S, Fryers PT. Evaluation of a disposable prism for applanation tonometry. Eye 2001; 15: 279-282.

14 Kaufmann C, Bachmann LM, Thiel MA. Comparison of dynamic contour tonometry with goldmann applanation tonometry. Invest Ophthalmol Vis Sci 2004; 45: 3118-3121.

15 Kotecha A, White ET, Shewry JM, Garway-Heath DF. The relative effects of corneal thickness and age on Goldmann applanation tonometry and dynamic contour tonometry. Br J Ophthalmol 2005; 89: 1572-1575.

16 Doyle A, Lachkar Y. Comparison of dynamic contour tonometry with goldman applanation tonometry over a wide range of central corneal thickness. J Glaucoma 2005; 14: 288-292.

17 Siganos DS, Papastergiou GI, Moedas C. Assessment of the Pascal dynamic contour tonometer in monitoring intraocular pressure in unoperated eyes and eyes after LASIK. J Cataract Refract Surg 2004; 30: 746-751.

18 Kaufmann C, Bachmann LM, Thiel MA. Intraocular pressure measurements using dynamic contour tonometry after laser in situ keratomileusis. Invest Ophthalmol Vis Sci 2003; 44: 3790-3794.

19 Schneider E, Grehn F. Intraocular pressure measurement - comparison of dynamic contour tonometry and Goldmann applanation tonometry. J Glaucoma 2006; 15: 2-6. 
20 Ogbuehi KC. Assessment of the accuracy and reliability of the Topcon CT80 non-contact tonometer. Clin Exp Optom 2006; 89: 310-314.

21 Goldmann H. Factors influencing intraocular pressure. Bibl Ophthalmol 1970; 81: 97-105.

22 Bhan A, Browning AC, Shah S, Hamilton R, Dave D, Dua HS. Effect of corneal thickness on intraocular pressure measurements with the pneumotonometer, Goldmann applanation tonometer and Tono-Pen. Invest Ophthalmol Vis Sci 2002; 43: 1389-1392.

23 Tonnu PA, Ho T, Newson T, El Sheikh A, Sharma K, White E et al. The influence of central corneal thickness and age on intraocular pressure measured by pneumotonometry, non-contact tonometry, the Tono-Pen $\mathrm{XL}$, and Goldmann applanation tonometry. Br J Ophthalmol 2005; 89: 851-854.

24 Yagci R, Eksioglu U, Midillioglu I, Yalvac I, Altiparmak E, Duman S. Central corneal thickness in primary open angle glaucoma, pseudoexfoliative glaucoma, ocular hypertension, and normal population. Eur J Ophthalmol 2005; 15: 324-328.
25 Kniestedt C, Nee M, Stamper RL. Dynamic contour tonometry: a comparative study on human cadaver eyes. Arch Ophthalmol 2004; 122: 1287-1293.

26 Francis BA, Hsieh A, Lai MY, Chopra V, Pena F, Azen S, et al., Los Angeles Latino Eye Study Group. Effects of corneal thickness, corneal curvature, and intraocular pressure level on Goldmann applanation tonometry and dynamic contour tonometry. Ophthalmology 2007; 114: 20-26.

27 Hoffmann EM, Grus FH, Pfeiffer N. Intraocular pressure and ocular pulse amplitude using dynamic contour tonometry and contact lens tonometry. BMC Ophthalmol 2004; 4: 4.

28 Kamppeter BA, Jonas JB. Dynamic contour tonometry for intraocular pressure measurement. Am J Ophthalmol 2005; 140: $318-320$.

29 Barleon L, Hoffmann EM, Berres M, Pfeiffer N, Grus FH. Comparison of dynamic contour tonometry and Goldmann applanation tonometry in glaucoma patients and healthy subjects. Am J Ophthalmol 2006; 142: 583-590. 